\title{
Modelling NO Reductase Reaction via Heme-NO and NO Coupling Activated by Lewis Acid
}

\section{YONG ZHANG}

\section{Department of Chemistry and Chemical Biology}

Stevens Institute of Technology
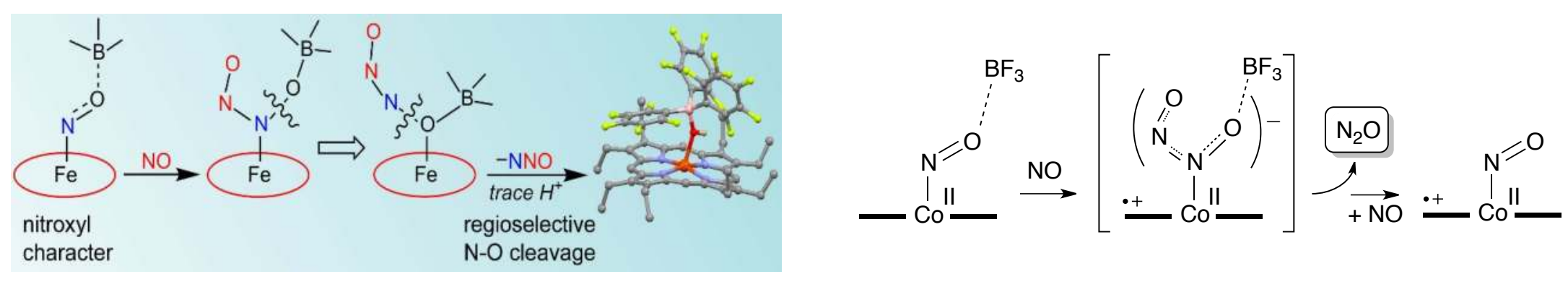


\section{Nitric oxide reductase (NOR)}

* Biological denitrification

- Inactivation of NO from host immune system by bacteria

$2 \mathrm{NO}+2 \mathrm{H}^{+}+2 \mathrm{e}^{-} \rightarrow \mathrm{N}_{2} \mathrm{O}+\mathrm{H}_{2} \mathrm{O}$

NO coupling mechanism?

(Por)Fe $\mathrm{II}^{\mathrm{II}}$ NO)

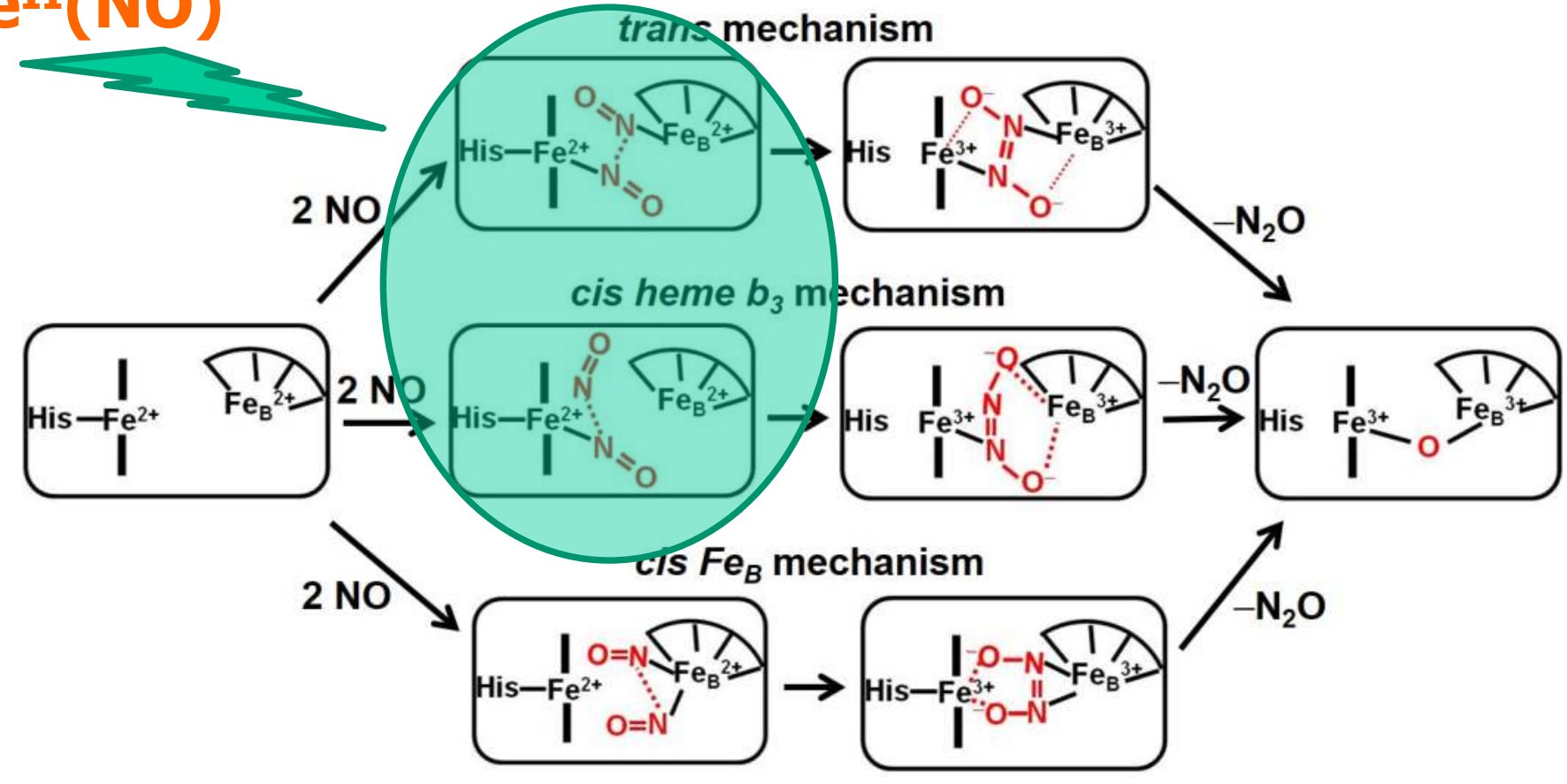

S. Chakraborty, J. Reed, M. Ross, M. J. Nilges, I. D. Petrik, S. Ghosh, S. Hammes-Schiffer, J. T. Sage, Y. Zhang, C. E. Schulz, Y. Lu. Angew. Chem. Int. Ed. 53, 2417 (2014); Richter-Addo et al. J. Am. Chem. Soc. 131, 2460 (2009); Richter-Addo et al. J. Am. Chem. Soc. 140, 4204 (2018) 


\section{NO Coupling: Expt $\rightarrow$ Calc}

$(\mathrm{OEP}) \mathrm{Fe}\left({ }^{15} \mathrm{NO}\right)+\mathrm{NO} \rightleftharpoons(\mathrm{OEP}) \mathrm{Fe}(\mathrm{NO})+{ }^{15} \mathrm{NO}$

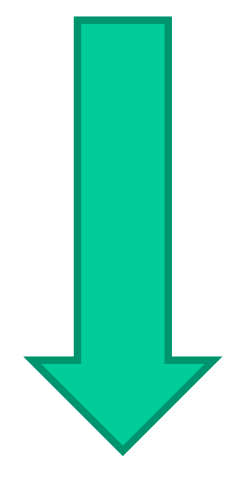

NO coupling energies

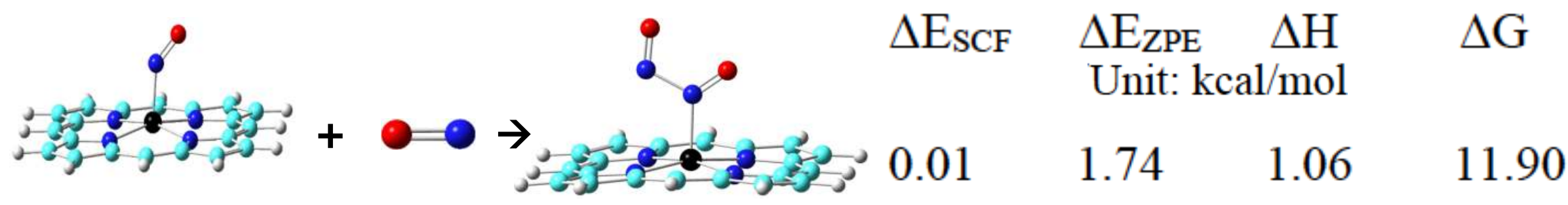

Heme-NO alone does not enable coupling 


\section{NO Coupling: Calc $\rightarrow$ Expt}

\section{NO coupling energies}

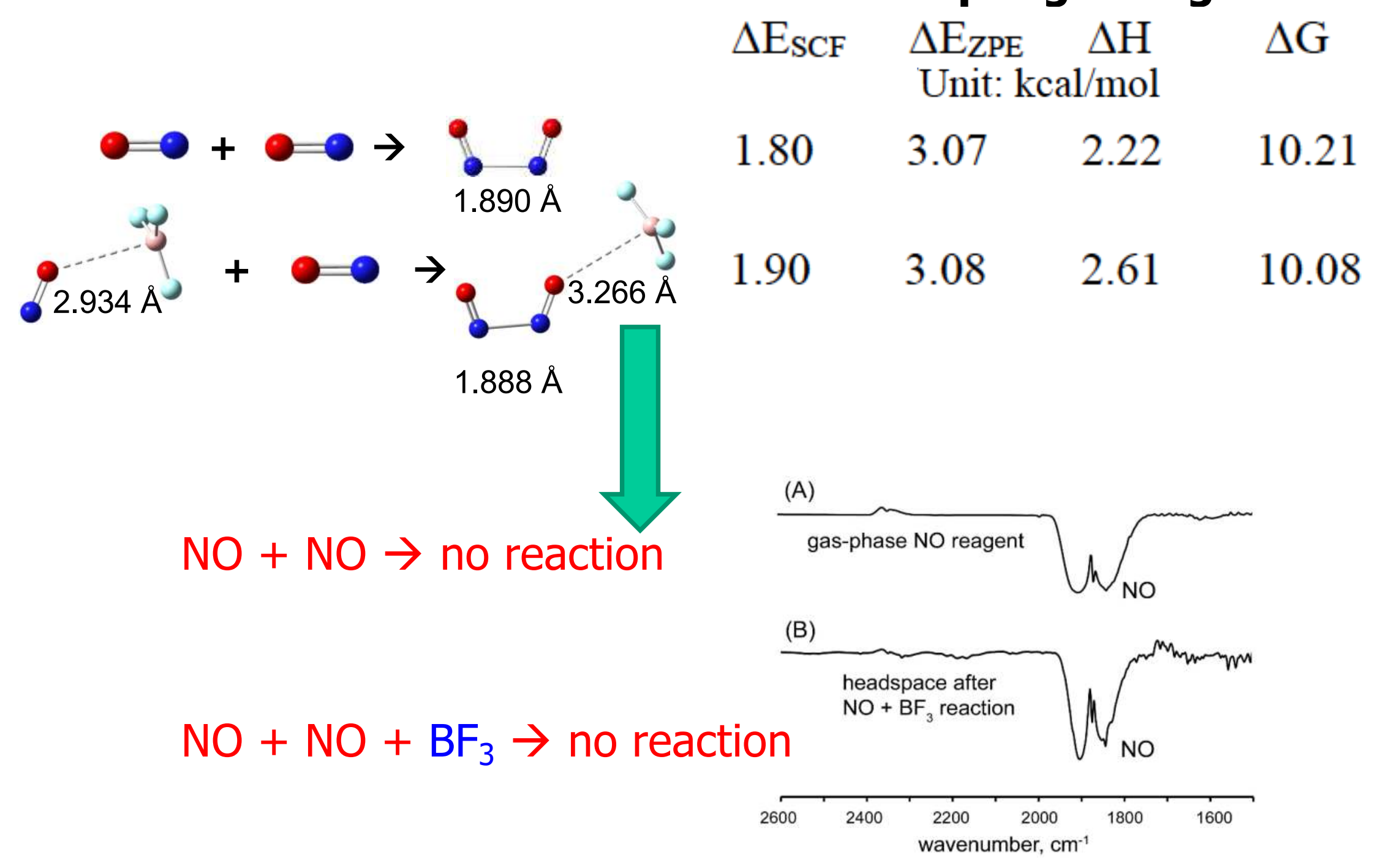

$\mathrm{BF}_{3}$ alone does not enable coupling

Abucayon, Khade, Powell, Zhang, Richter-Addo. J. Am. Chem. Soc. 140, 4204 (2018) 


\section{NO Coupling: Expt $\rightarrow$ Calc}
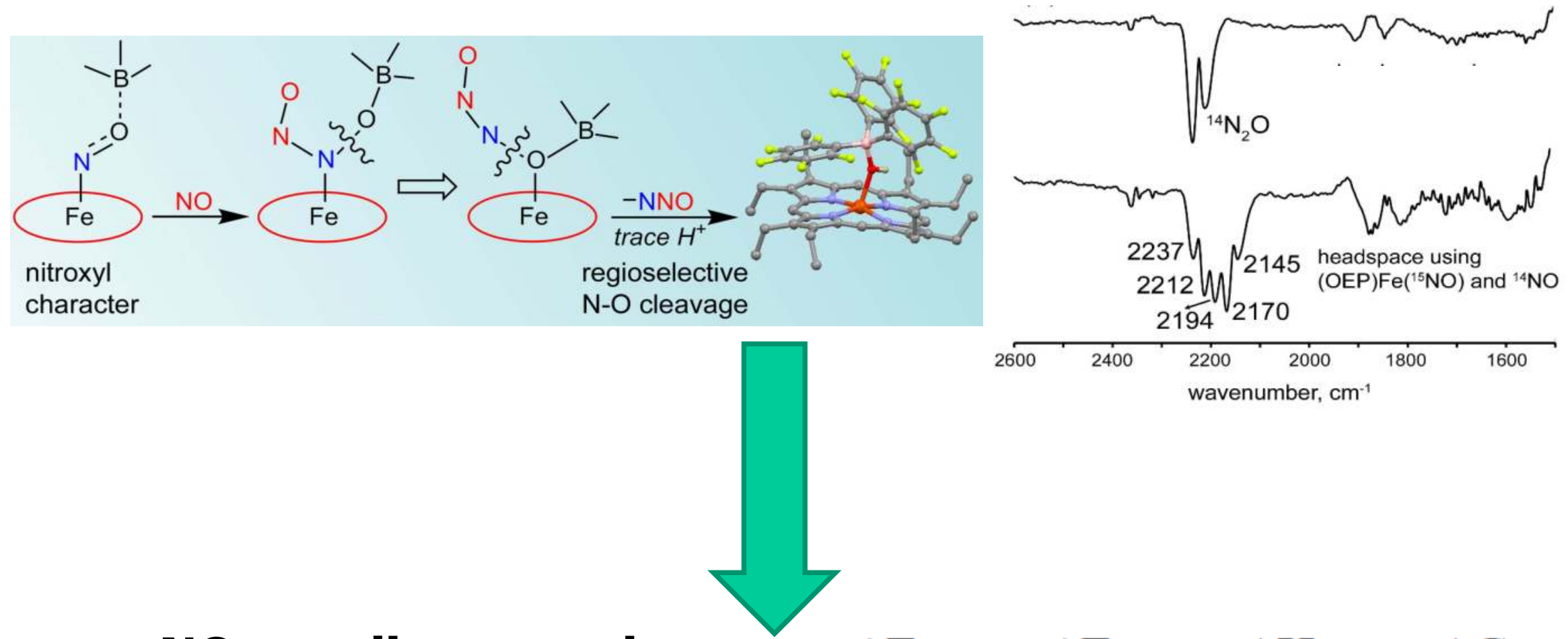

\section{NO coupling energies}
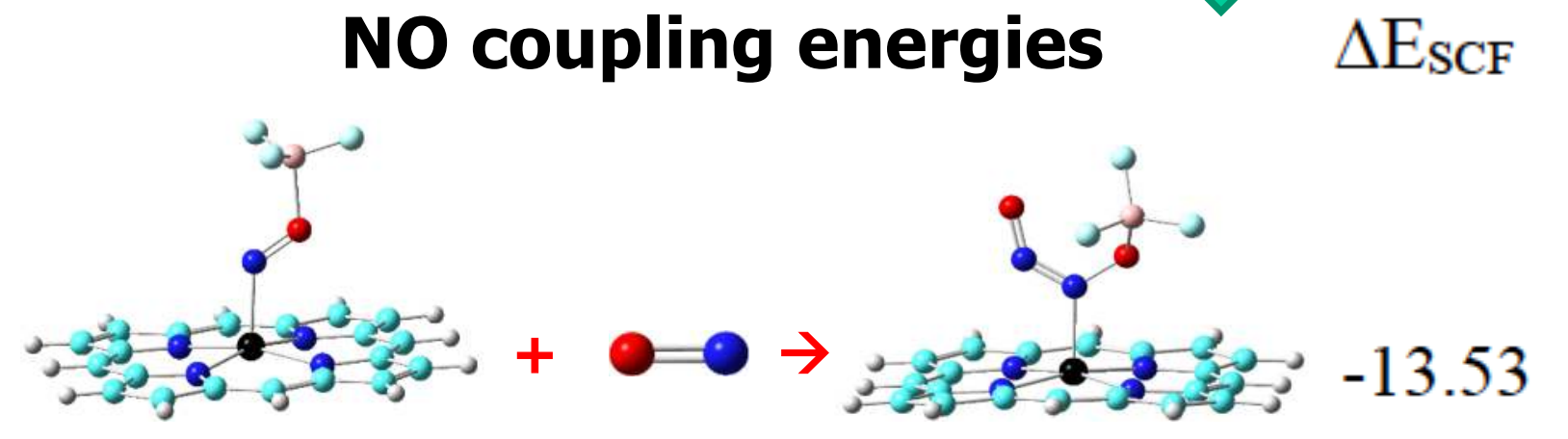

$\underset{\text { Unit: } \mathrm{kcal} / \mathrm{mol}}{\Delta \mathrm{mol}}$

$\Delta \mathrm{G}$

Heme-NO and $\mathrm{BF}_{3}$ together enable coupling

Abucayon, Khade, Powell, Zhang, Richter-Addo. J. Am. Chem. Soc. 140, 4204 (2018) 


\section{Origin of Synergistic NO Coupling}

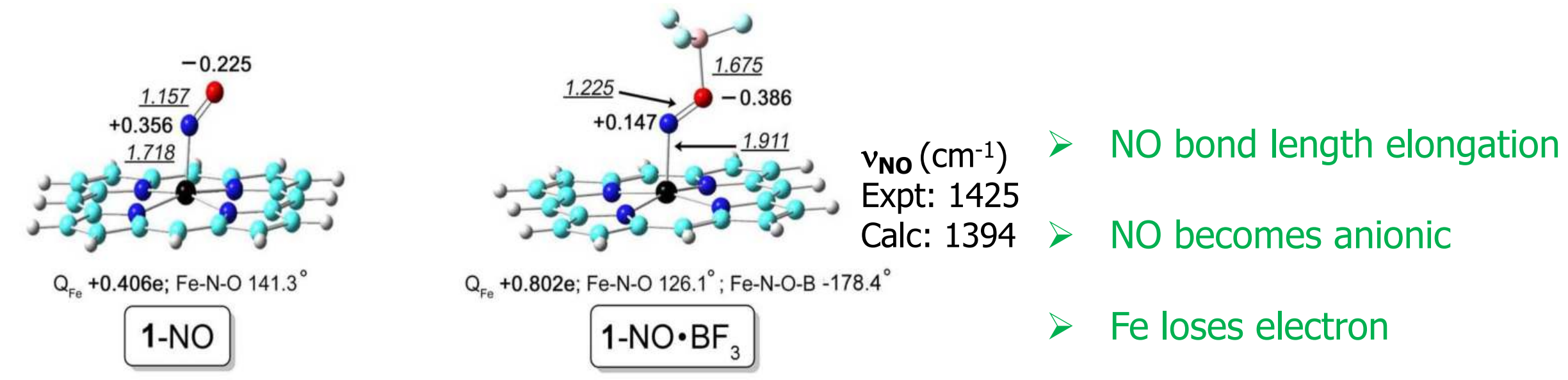

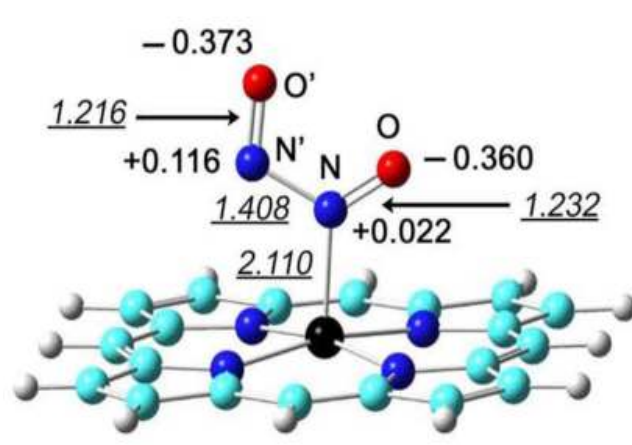

$\mathrm{Q}_{\mathrm{Fe}}+0.958 \mathrm{e} ; \mathrm{Fe}-\mathrm{N}-\mathrm{O} 124.3^{\circ}$

$1-(\mathrm{NO})_{2}$

Repulsive NO...NO

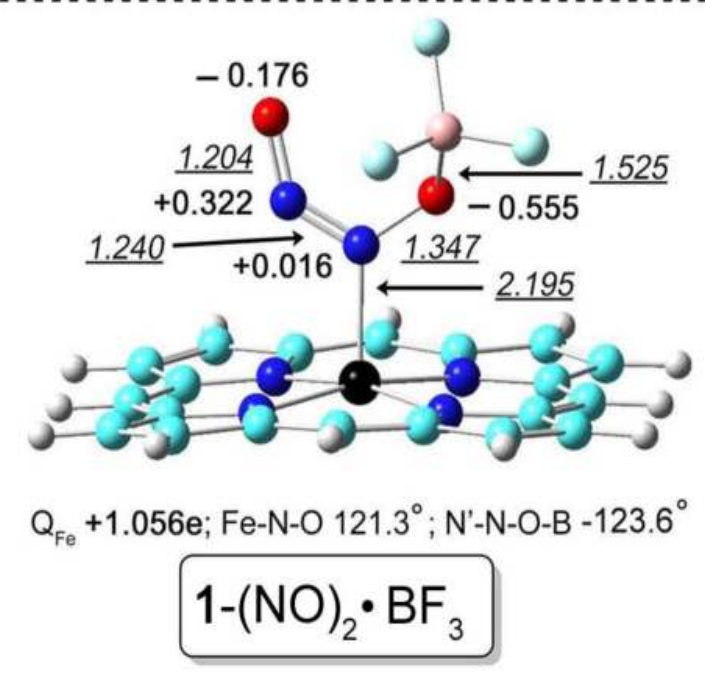

attractive NO...NO
$\mathrm{BF}_{3}$ pulls $e_{1} \mathrm{Fe}$ (Por) donates $e$

$>$ Bound NO becomes more anionic with enhanced NO bond length elongation

$>$ Incoming $\mathrm{NO}$ becomes cationic to favor NO coupling with shortened $\mathrm{N}$ $\mathrm{N}$ bond length 


\section{Spin state effect}

Table S1. Spin Densities (Unit: e) and Energies (Unit: kcal/mol) of Different NO Coupled Isomers

\begin{tabular}{|c|c|c|c|c|c|c|c|c|}
\hline Systems & $\mathrm{S}$ & $\rho_{\alpha \beta}{ }^{\mathrm{Fe}}$ & $\rho_{\alpha \beta}{ }^{\mathrm{NO}}$ & $\rho_{\alpha \beta}{ }^{\mathrm{N}^{\prime} \mathrm{O}^{\prime}}$ & $\Delta \mathrm{E}_{\mathrm{SCF}}$ & $\Delta \mathrm{E}_{\mathrm{ZPE}}$ & $\Delta \mathrm{H}$ & $\Delta \mathrm{G}$ \\
\hline \multirow[t]{2}{*}{$(\mathrm{NO})_{2}$} & 0 & & 0.00 & 0.00 & 0.00 & 0.00 & 0.00 & 0.00 \\
\hline & 1 & & 1.000 & 1.000 & 0.24 & -0.24 & -0.20 & -0.89 \\
\hline \multirow[t]{2}{*}{$\mathrm{ONNO} \cdots \mathrm{BF}_{3}$} & 0 & & 0.00 & 0.00 & 0.00 & 0.00 & 0.00 & 0.00 \\
\hline & 1 & & 1.023 & 0.975 & 0.56 & -0.09 & 0.12 & -2.61 \\
\hline \multirow[t]{3}{*}[\mathrm{Fe}(\mathrm{P})(\mathrm{NO})_{2}]{} & 0 & 1.195 & -0.808 & -0.247 & 0.00 & 0.00 & 0.00 & 0.00 \\
\hline & 1 & 1.322 & 0.475 & 0.364 & 4.28 & 3.82 & 4.06 & 2.60 \\
\hline & 1 & 2.905 & -0.498 & -0.311 & -4.79 & -5.30 & -4.98 & -6.87 \\
\hline \multirow[t]{3}{*}[\mathrm{Fe}(\mathrm{P})(\mathrm{ONN}-\cdots\mathrm{BF}_{3})]{} & 0 & 1.166 & -0.119 & -0.873 & 0.00 & 0.00 & 0.00 & 0.00 \\
\hline & 1 & 1.170 & -0.063 & 1.000 & -0.65 & -0.54 & -0.62 & -1.07 \\
\hline & 1 & 2.989 & 0.053 & -0.928 & -14.27 & -14.60 & -14.45 & -16.09 \\
\hline & & & & & & & & \\
\hline & & & & \multicolumn{5}{|c|}{ NO $(S=1 / 2) \uparrow \uparrow \mathbf{N O}(S=1 / 2)$} \\
\hline
\end{tabular}

All prefer triplet
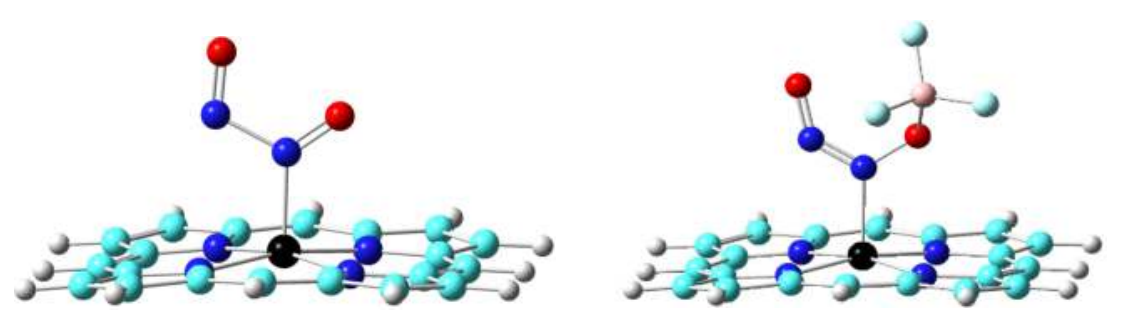

$\mathrm{Fe}^{\mathrm{III}}(\mathrm{S}=3 / 2) \uparrow \downarrow(\mathrm{NO})_{2}^{-}(\mathrm{S}=1 / 2)$

Fe is oxidized

Abucayon, Khade, Powell, Zhang, Richter-Addo. J. Am. Chem. Soc. 140, 4204 (2018) 


\section{Conformation effect}

Table S3. Relative Energies of Different Conformations (Units: kcal $/ \mathrm{mol}$ )

\begin{tabular}{|c|c|c|c|c|c|c|}
\hline Compound & Conformation & Structure & $\Delta \mathrm{E}_{\mathrm{SCF}}$ & $\Delta \mathrm{E}_{\mathrm{ZPE}}$ & $\Delta \mathrm{H}$ & $\Delta \mathrm{G}$ \\
\hline \multirow[t]{2}{*}{$(\mathrm{NO})_{2}$} & cis & : : & 0.00 & 0.00 & 0.00 & 0.00 \\
\hline & trans & 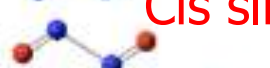 & 0.86 & 0.72 & 0.79 & 0.75 \\
\hline \multirow[t]{3}{*}[\mathrm{Fe}(\mathrm{P})(\mathrm{NO}\cdots\mathrm{BF}_{3})]{} & cis & & 0.00 & 0.00 & 0.00 & 0.00 \\
\hline & trans & 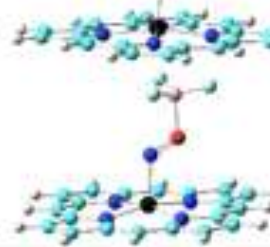 & $\begin{array}{l}\text { eferred } \\
0.11\end{array}$ & 0.31 & -0.21 & 1.08 \\
\hline & & & $\begin{array}{l}\left.\mathrm{n}^{-1}\right) \\
425 \\
\text { is): } 139 \\
\text { rans): } 1\end{array}$ & & & \\
\hline
\end{tabular}

Abucayon, Khade, Powell, Zhang, Richter-Addo. J. Am. Chem. Soc. 140, 4204 (2018) 


\section{NO Coupling: Co}

$(\mathrm{OEP}) \mathrm{Co}(\mathrm{NO})+\operatorname{excess}{ }^{15} \mathrm{NO} \rightarrow(\mathrm{OEP}) \mathrm{Co}\left({ }^{15} \mathrm{NO}\right)+\mathrm{NO}$ $(\mathrm{OEP}) \mathrm{Co}(\mathrm{NO})+\mathrm{BF}_{3} \cdot \mathrm{OEt}_{2}$
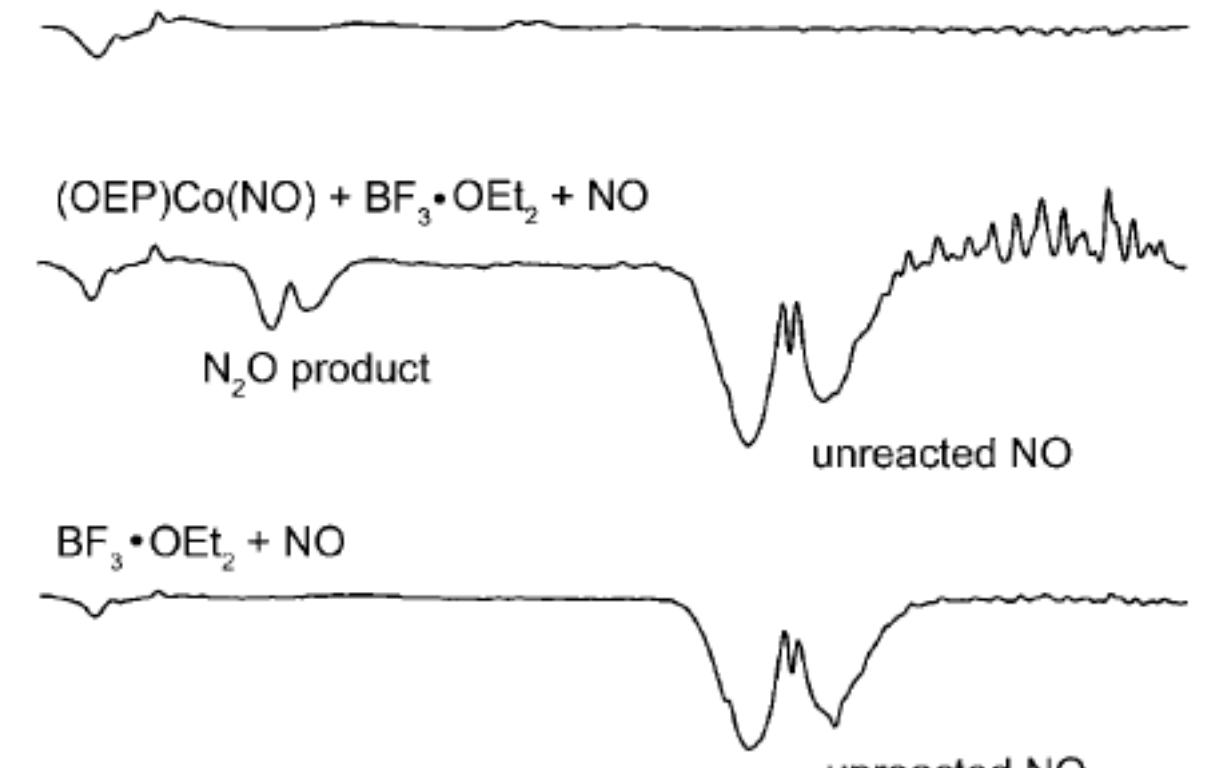

unreacted NO

$2400 \quad 2000$
wavenumber, $\mathrm{cm}^{-1}$

Synergistic heme-NO and NO coupling activated by Lewis acid

Abucayon, Khade, Powell, Zhang, Richter-Addo. Angew. Chem. Int. Ed. 58, 18598 (2019) 


\section{Origin of Synergistic NO Coupling}

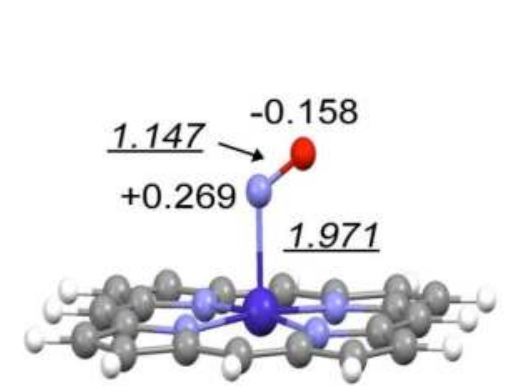

$\mathrm{Q}_{\mathrm{Co}}+0.608 \mathrm{e} ; \mathrm{Q}_{\mathrm{p}}-0.720 \mathrm{e}$

$\rho^{\mathrm{Co}}+0.895 ; \rho^{\mathrm{p}}-0.064$;

$\angle \mathrm{Co}-\mathrm{N}-\mathrm{O} 122.5^{\circ}$

1-NO
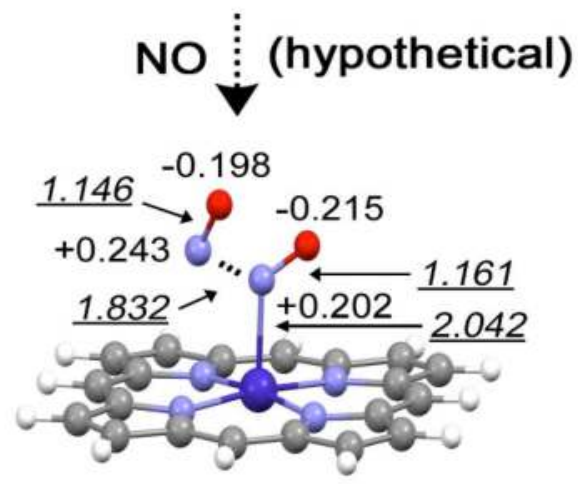

$\mathrm{Q}_{\mathrm{Co}}+0.665 \mathrm{e} ; \mathrm{Q}_{\mathrm{p}}-0.697 \mathrm{e}$;

$\rho^{\mathrm{Co}}+0.899 ; \rho^{\mathrm{p}}-0.111$;

$\angle \mathrm{Co}-\mathrm{N}-\mathrm{O} 126.4^{\circ}$

$2-(\mathrm{NO})_{2}$

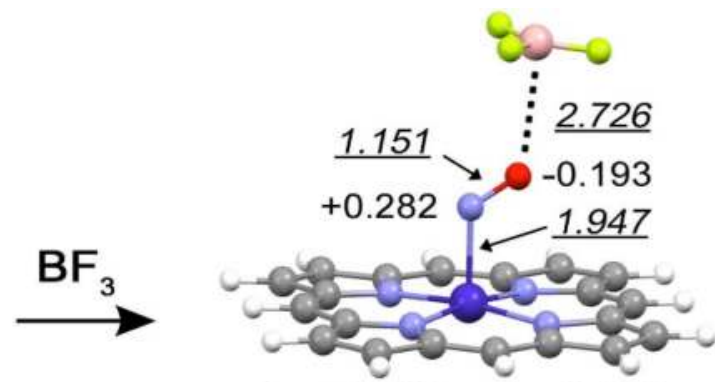

$\mathrm{Q}_{\mathrm{Co}}+0.608 \mathrm{e} ; \mathrm{Q}_{\mathrm{p}}-0.690 \mathrm{e}$

$\rho^{\mathrm{Co}}+0.876 ; \rho^{\mathrm{P}}-0.067$;

$\angle \mathrm{Co}-\mathrm{N}-\mathrm{O} 121.8^{\circ} ; \angle \mathrm{Co}-\mathrm{N}-\mathrm{O}-\mathrm{B} 179.0^{\circ}$

$1-\mathrm{NO} \cdot \mathrm{BF}_{3}$
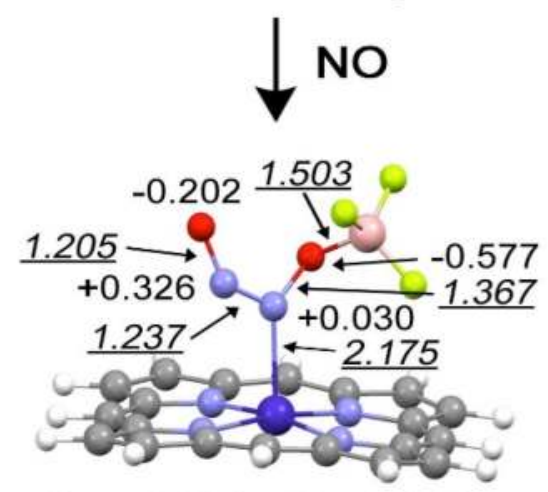

$\mathrm{Q}_{\mathrm{Co}}+0.746 \mathrm{e} ; \mathrm{Q}_{\mathrm{p}}+0.061 \mathrm{e}$;

$\rho^{\mathrm{Co}}+0.984 ; \rho^{\rho}-1.005 ;$

$\angle \mathrm{Co}-\mathrm{N}-\mathrm{O} 127.0^{\circ} ; \angle \mathrm{Co}-\mathrm{N}-\mathrm{O}-\mathrm{B}-101.4^{\circ}$

$2-(\mathrm{NO})_{2} \cdot \mathrm{BF}_{3}$
$>$ NO bond length slight elongation

$>$ NO becomes less cationic

> Porphyrin loses electron

$\mathrm{BF}_{3}$ pulls $e_{1} \mathrm{Co}$ (Por) donates $e$

$>$ Bound NO becomes more anionic with enhanced NO bond length elongation

$>$ Incoming $\mathrm{NO}$ becomes more cationic to favor attractive NO-NO coupling with shortened $\mathrm{N}-\mathrm{N}$ bond length

Abucayon, Khade, Powell, Zhang, Richter-Addo. Angew. Chem. Int. Ed. 58, 18598 (2019) 


\section{Lewis acid effect on $M(P)\left(\mathrm{NO}_{\text {...BF }}\right)$ : Co vs. Fe}

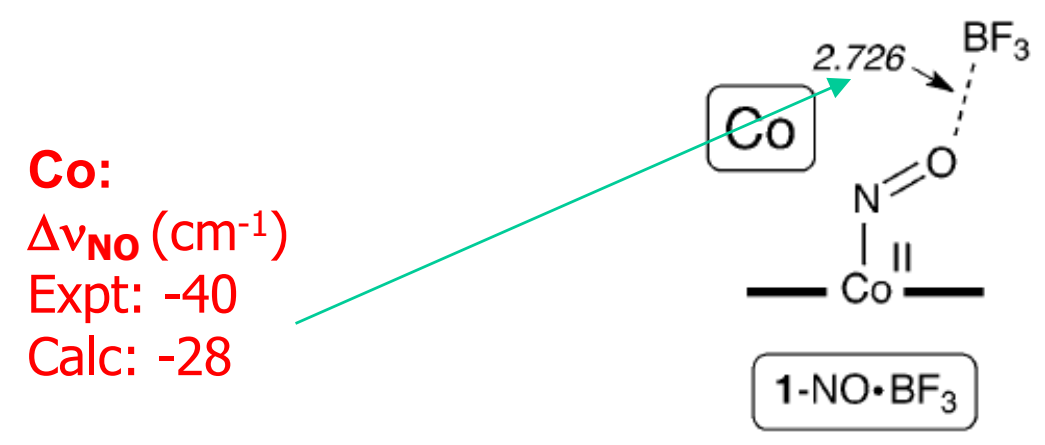

Fe:

$\Delta v_{\text {NO }}\left(\mathrm{cm}^{-1}\right)$

Expt: -241

Calc: -272

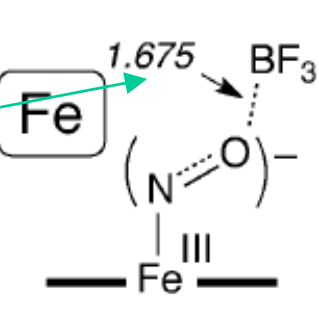

Abucayon, Khade, Powell, Zhang, Richter-Addo. Angew. Chem. Int. Ed. 58, 18598 (2019) 


\section{Lewis acid effect on $M(P)\left(N_{0} . . . B_{3}\right)$ : Co vs. Fe}

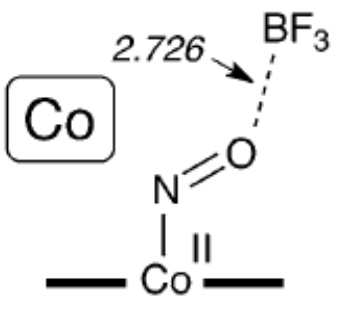

$1-\mathrm{NO} \cdot \mathrm{BF}_{3}$
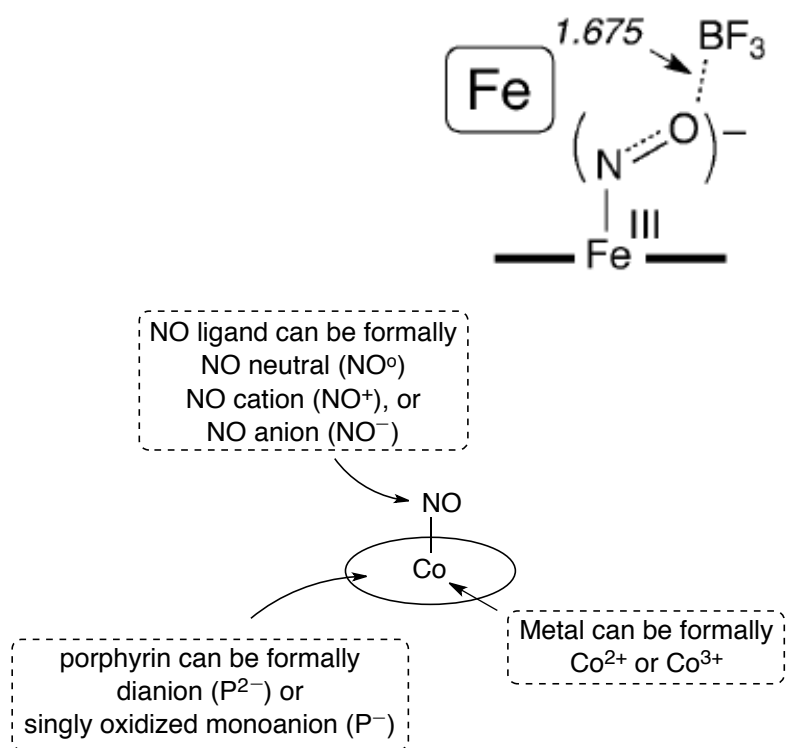

$>$ Co essentially keeps CoII state with charge increase only by $+0.138 \mathrm{e}$

$>$ Fe is largely oxidized with a significant charge increase of +0.650 e
Table S4. Studied Fragment Spin States for $(\mathrm{P}) \mathrm{Co}\left(\mathrm{NO}^{\cdots} \mathrm{BF}_{3}\right)(S=0)$

\begin{tabular}{llll}
\hline State & $\mathrm{Co}$ & $\mathrm{P}$ & $\mathrm{NO}$ \\
\hline N-II-1 & $\mathrm{Co}^{2+}(S=1 / 2)$ & $\mathrm{P}^{2-}(S=0)$ & $\mathrm{NO}(S=-1 / 2)$ \\
N-II-2 & $\mathrm{Co}^{2+}(S=1 / 2)$ & $\mathrm{P}^{-}(S=-1 / 2)$ & $\mathrm{NO}^{-}(S=0)$ \\
N-II-3 & $\mathrm{Co}^{2+}(S=-1 / 2)$ & $\mathrm{P}^{-}(S=-1 / 2)$ & $\mathrm{NO}^{-}(S=1)$ \\
N-III-1 & $\mathrm{Co}^{3+}(S=0)$ & $\mathrm{P}^{2-}(S=0)$ & $\mathrm{NO}^{-}(S=0)$ \\
N-III-2 & $\mathrm{Co}^{3+}(S=1)$ & $\mathrm{P}^{2-}(S=0)$ & $\mathrm{NO}^{-}(S=-1)$ \\
\hline
\end{tabular}

Table 1: Selected fragment spin states studied, and the spin densities and relative electronic energies of the optimized geometries. ${ }^{[a]}$

\begin{tabular}{|c|c|c|c|c|c|c|c|c|c|c|c|}
\hline System ${ }^{[b]}$ & spin & state $e^{[c]}$ & Co & $\mathrm{P}$ & axial $(\mathrm{NO})_{n}$ & $\rho_{\alpha \beta} \mathrm{Co}^{[d]}$ & $\rho_{\alpha \beta} \mathrm{P}^{[\mathrm{d}]}$ & $\rho_{a \beta} \mathrm{NO}^{[d]}$ & $\rho_{\alpha \beta} N^{\prime} O^{\prime[d]}$ & $\rho_{\alpha \beta} B F_{3}^{[d]}$ & $\Delta E_{\mathrm{SCF}}^{[\text {e] }}$ \\
\hline $1-\mathrm{NO} \cdot \mathrm{BF}_{3}$ & 0 & $\mathrm{~N}-\mathrm{II}-1$ & $\mathrm{Co}^{2+}\left(S={ }^{1} / 2\right)$ & $\mathrm{P}^{2-}(S=0)$ & $\mathrm{NO}(S=-1 / 2)$ & 0.876 & -0.067 & -0.802 & & -0.067 & 0.00 \\
\hline & 0 & N-III-1 & $\mathrm{Co}^{3+}(\mathrm{S}=0)$ & $\mathrm{P}^{2-}(S=0)$ & $\mathrm{NO}^{-}(\mathrm{S}=0)$ & 0.000 & 0.000 & 0.000 & & 0.000 & 4.38 \\
\hline
\end{tabular}




\section{Product feature}

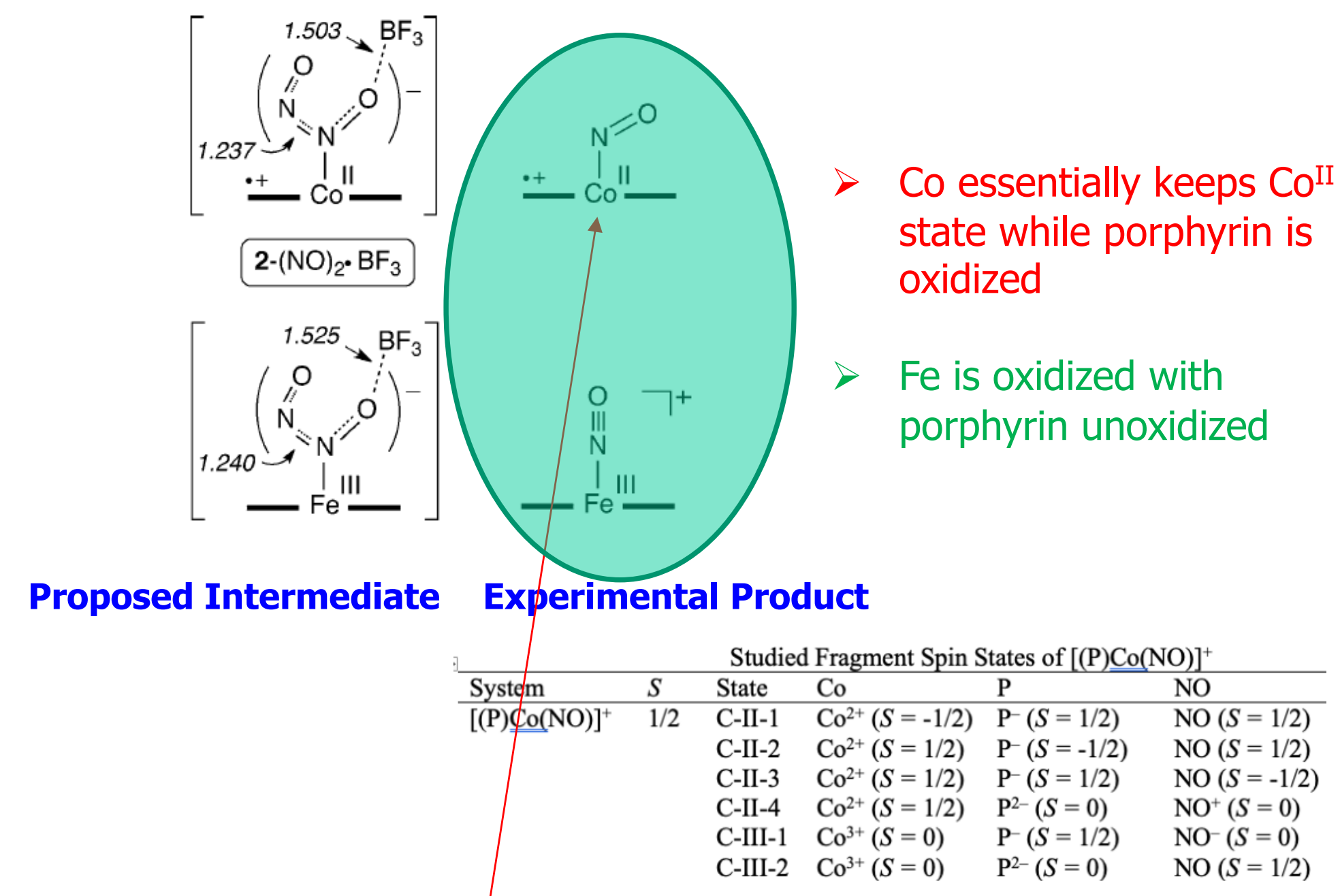

Table 1: Selected fragment spin states studied, and the spin den $\$$ ities and relative electronic energies of the optimized geometries. ${ }^{[a]}$

\begin{tabular}{|c|c|c|c|c|c|c|c|c|c|c|c|}
\hline System ${ }^{[b]}$ & spin & state $^{[c]}$ & Co & $P$ & axial $(\mathrm{NO})_{n}$ & $\rho_{\alpha \beta} \mathrm{Co}^{[\mathrm{d}]}$ & $\rho_{\alpha \beta} \mathrm{P}^{[\mathrm{d}]}$ & $\rho_{\alpha \beta} \mathrm{NO}^{[\mathrm{d}]}$ & $\rho_{\alpha \beta} \mathrm{N}^{\prime} \mathrm{O}^{\prime[d]}$ & $\rho_{\alpha \beta} \mathrm{BF}_{3}^{[d]}$ & $\Delta E_{\mathrm{SCF}}^{\left[{ }^{[e]}\right.}$ \\
\hline$\left[1-\mathrm{NO}^{+}\right.$ & $1 / 2$ & C-II-1] & $\mathrm{Co}^{2+}(\mathrm{S}=-1 / 2)$ & $\mathrm{P}^{-}(\mathrm{S}=1 / 2)$ & $\mathrm{NO}(S=1 / 2)$ & -0.900 & 1.056 & 0.844 & & & 0.00 \\
\hline & $1 / 2$ & C-III-1 & $\mathrm{Co}^{3+}(\mathrm{S}=0)$ & $P^{-}(S=1 / 2)$ & $\mathrm{NO}^{-}(\mathrm{S}=0)$ & 0.014 & 0.989 & -0.003 & & & 7.09 \\
\hline
\end{tabular}




\section{Intermediate feature}

$>$ Co essentially keeps CoII state while porphyrin is oxidized

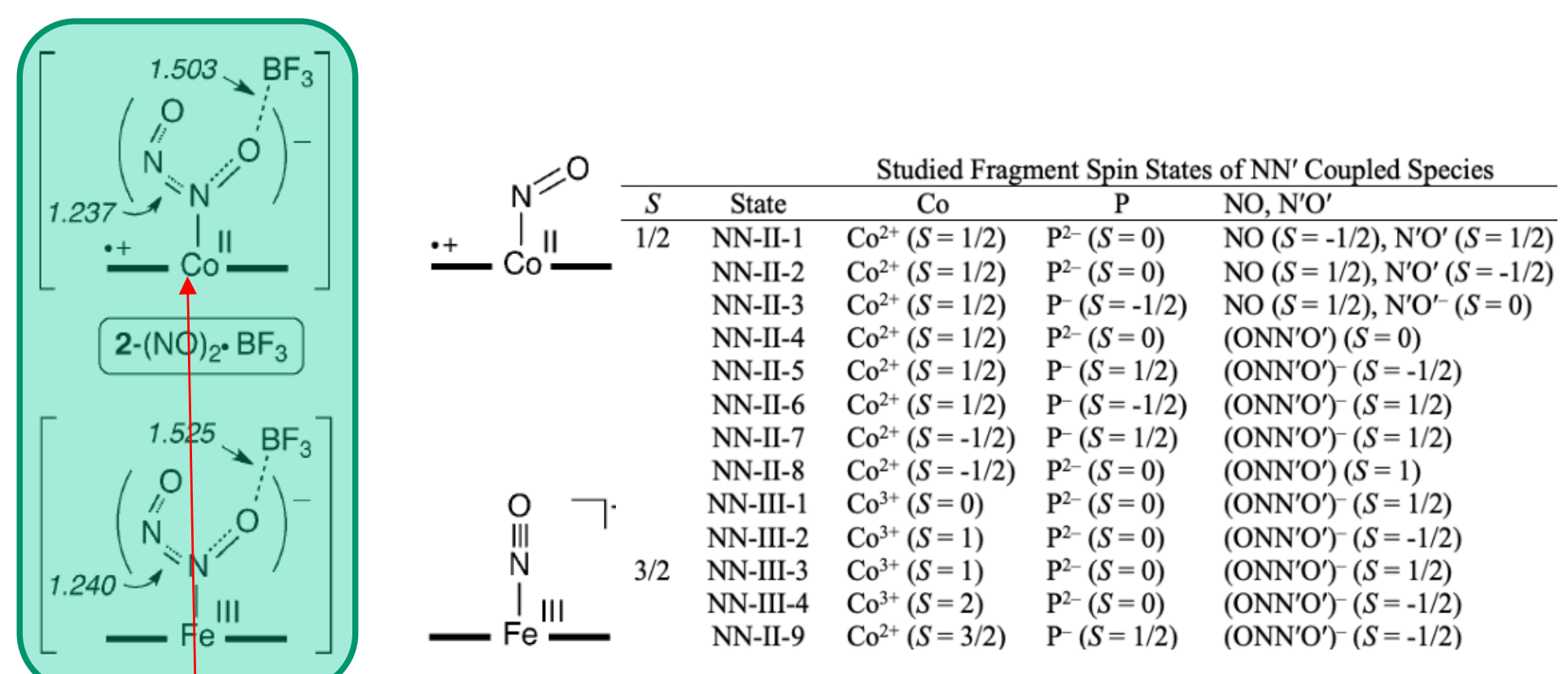

Table 1: Selected fragment spin states studied, and the spin densities and relative electronic energies of the optimized geometries. ${ }^{[a]}$

\begin{tabular}{|c|c|c|c|c|c|c|c|c|c|c|c|}
\hline System $^{[b]}$ & spin & state $e^{[c]}$ & Co & $\mathrm{P}$ & $\operatorname{axial}(\mathrm{NO})_{n}$ & $\rho_{\alpha \beta} \mathrm{Co}^{[d]}$ & $\rho_{\alpha \beta} \mathrm{P}^{[\mathrm{d}]}$ & $\rho_{\alpha \beta} \mathrm{NO}^{[\mathrm{d}]}$ & $\rho_{\alpha \beta} \mathrm{N}^{\prime} \mathrm{O}^{\prime[d]}$ & $\rho_{\alpha \beta} \mathrm{BF}_{3}{ }^{[\mathrm{d}]}$ & $\Delta E_{\mathrm{SCF}}^{[\mathrm{e}]}$ \\
\hline \multirow[t]{6}{*}{$2-(\mathrm{NO})_{2} \cdot \mathrm{BF}_{3}$} & $1 / 2$ & NN-II-6 & $\operatorname{Co}^{2+}(S=1 / 2)$ & $\mathrm{P}^{-}\left(S=-^{1} / 2\right)$ & $(\mathrm{NO})_{2}{ }^{-}(S=1 / 2)$ & 0.984 & -1.005 & 0.041 & 0.980 & 0.001 & 0.00 \\
\hline & & NN-II-7 & $\mathrm{Co}^{2+}(\mathrm{S}=-1 / 2)$ & $\mathrm{P}^{-}(S=1 / 2)$ & $(\mathrm{NO})_{2}{ }^{-}(\mathrm{S}=1 / 2)$ & -0.956 & 1.000 & 0.000 & 0.948 & 0.008 & 0.36 \\
\hline & $1 / 2$ & NN-III-I & $\mathrm{Co}^{\circ}(S=0)$ & $p^{2}(S=0)$ & $(\mathrm{NO})_{2} \quad(S=1 / 2)$ & -0.010 & 0.035 & 0.036 & 0.932 & 0.006 & 1.84 \\
\hline & $1 / 2$ & $\mathrm{NN}-\mathrm{III}-2$ & $\mathrm{Co}^{3+}(\mathrm{S}=1)$ & $\mathrm{P}^{2-}(S=0)$ & $(\mathrm{NO})_{2}{ }^{-}(\mathrm{S}=-1 / 2)$ & 1.964 & -0.087 & 0.039 & -0.911 & -0.006 & 1.51 \\
\hline & $3 / 2$ & NN-III-3 & $\mathrm{Co}^{3+}(S=1)$ & $\mathrm{P}^{2-}(S=0)$ & $(\mathrm{NO})_{2}{ }^{-}(S=1 / 2)$ & 1.964 & -0.051 & 0.095 & 0.990 & 0.002 & 0.69 \\
\hline & $3 / 2$ & NN-II-9 & $\mathrm{Co}^{2+}(S=3 / 2)$ & $\mathrm{P}^{-}(S=1 / 2)$ & $(\mathrm{NO})_{2}{ }^{-}(\mathrm{S}=-1 / 2)$ & 2.780 & 1.163 & -0.018 & -0.923 & -0.003 & 1.25 \\
\hline
\end{tabular}

Abucayon, Khade, Powell, Zhang, Richter-Addo. Angew. Chem. Int. Ed. 58, 18598 (2019) 


\section{Conclusions}

$>$ For nitric oxide reductase (NOR) model reactions, calculations reproduced the difficulty in direct heme-NO and NO coupling, and predicted that the observed coupling occurred in the presence of Lewis acid is due to the synergistic effect of both heme and Lewis acid, which was supported by further control experiments.

$>$ This synergistic effect on $\mathrm{N}-\mathrm{N}$ coupling was found to originate from cooperation of heme donating electron and Lewis acid attracting electron, which helps understand the importance of co-existence of both heme and non-heme (possible Lewis acid) sites in native NOR.

$>$ Both Fe and Co containing heme models can effect such coupling, and calculations support the significantly different experimental results: 1) Fe is oxidized with porphyrin unoxidized, while Co is unoxidized and porphyrin is oxidized; 2) Heme-NO and $\mathrm{BF}_{3}$ interaction is much stronger in the case of Fe than Co. 


\section{Acknowledgments}

Stevens Institute of Technology

Rahul Khade

University of Oklahoma

E. G. Abucayon

D. R. Powell

G. Richter-Addo

$\Delta$ National Institutes of Health

NIH grant GM-085774 